\title{
PEDIATRIC SCRUB TYPHUS WITH MENINGOENCEPHALITIS
}

Swati M. Gadappa ${ }^{1}$, Deepika Chopra²

\section{HOW TO CITE THIS ARTICLE:}

Swati M. Gadappa, Deepika Chopra. "Pediatric Scrub Typhus with Meningoencephalitis". Journal of Evolution of Medical and Dental Sciences 2014; Vol. 3, Issue 60, November 10; Page: 13477-13480,

DOI: $10.14260 /$ jemds/2014/3797

ABSTRACT: BACKGROUND: Pediatric Scrub typhus is under-diagnosed but life threatening febrile illness which requires early treatment. CASE CHARACTERISTICS: A 2-year-old boy presenting with fever, maculopapular rash, hepatosplenomegaly, altered sensorium and hemiparesis. OUTCOME: He was diagnosed as Scrub Typhus with Meningoencephalitis. MESSAGE: Pediatricians should consider scrub typhus in children with hemorrhagic rash, fever and hepatosplenomegaly, even in the absence of eschar.

KEYWORDS: Scrub Typhus, Meningoencephalitis, Weil Felix Antigen.

INTRODUCTION: Scrub typhus is an acute febrile illness caused by Orientia tsutsugamushi and is transmitted through the bite of larval mites (chiggers) of the Trombiculidae family. The disease has been reported from many regions of Asia and the Pacific islands. ${ }^{1}$ Indian tick typhus (ITT) has been reported in adults in Pune district of Maharashtra, Himachal Pradesh, in a French traveler returning from India and in Haryana.

The infection manifests clinically as a nonspecific febrile illness often accompanied by headache, myalgia, nausea, vomiting, diarrhea, cough, or breathlessness. The pathognomic clinical sign of scrub typhus is "eschar" (40-50\%) which may be inconspicuous. Severity varies from subclinical illness to severe illness with multiple organ system involvement, which can be serious enough to be fatal, unless diagnosed early and treated. Scrub typhus is grossly under-diagnosed in children in India.

We report a child with fever, rash, meningoencephalitis with positive Weil-Felix test in Pune, Maharashtra.

CASE REPORT: 2year old male child, $2^{\text {nd }}$ issue of NCM, was bought to the hospital with fever since 8days, rash since 4days, irritability and poor feeding. Child had a non-conclusive CBC.

Mother had noted generalized erythematous, non-pruritic, non-evanescent maculopapular rash beginning from upper and lower limbs, further spreading over next two days over trunk, face, palms and soles. The rash evolved into hyperpigmented lesions. No eschar was noted. There was no history of similar illness in the family or surrounding area. Child stays in a distant village in Pune district and plays with pet dog and cattle at home. However accurate history of insect bite was not obtained.

Fever was moderate to high grade. Child had poor oral intake and increased somnolence sin but no h/o seizures, vomiting, neurodeficit, breathlessness, oliguria and jaundice. Parents noticed edema of both hands and feet on day5 of illness. Child was well immunized and was growing well till onset of illness. Development was normal for age.

On examination, GCS-E4M5V3 12/15.Pallor+. No mucosal/conjunctival involvement. No lymphadenopathy, icterus. Rash noted. B/L pedal edema grade1. Normotensive. Signs of meningeal 
irritation were noted. Child also had Hepatosplenomegaly [L7S9]. Fundus examination-Disc normal. Bilateral vasculitic retinal changes with choroidal\& retinal infarcts in right eye noted. CSF study was s/o meningoencephalitis with mononuclear predominance. Child was started on oral Doxycycline and IV Chloramphenicol, Ceftriaxone suspecting Scrub typhus.PCV transfusion given for Anemia [Hb-6.5g/dl]. RMT- negative.

On day 2 of admission, patient had generalized tonic seizure with post-ictal left hemiparesis. Sensorium remained depressed for about one week thereafter requiring $3 \% \mathrm{NaCl}$ infusion.

\begin{tabular}{|c|c|c|}
\hline Weil Felix Antigen & D10 & D21 \\
\hline OX K & $1: 80$ & $1: 80$ \\
\hline OX 19 & $1: 80$ & $1: 80$ \\
\hline OX 2 & $1: 160$ & $1: 40$ \\
\hline
\end{tabular}

CSF LATEX - Negative.PT, aPTT- normal. Child had transient anicteric hepatitis in $2^{\text {nd }}$ week of illness.

MRI BRAIN:? vasculitis/ rickettsial encephalopathy. EEG s/o encephalopathy.

By day 7, Hepatosplenomegaly gradually regressed. Rash resolved gradually.

Patient's sensorium though remained depressed [GCS-12/15]. Hence repeat MRI Brain was done which showed regression of diffusion restriction lesions and appearance of new enhancing lesions seen in periventricular region, left caudate nucleus \& dorsal medulla. Child had no further seizures or fever. Hemiparesis gradually improved. Rash gradually resolved. SGPT showed rising trend in $3^{\text {rd }}$ wk of illness. Child did not follow light. Ophthal- healing RE retina. Child was discharged on home based physiotherapy \& oral phenytoin. With regular physiotherapy he recovered completely with normal vision in the next 1year.Spotted fever is rare in children and presence of hemorrhagic rash with fever, hepatosplenomegaly should make one suspect spotted fever.

DISCUSSION: Rickettsial fever has been reported from various parts of the country. Pediatric scrub typhus, however, has not been reported from Western India.

In series by Vivekanandan et al; eschar was found in 23 out of the 50 cases. The common sites involved were axilla, groin and thorax. ${ }^{2}$ In other reports from India very few patients were found to have eschar.3,4 But, eschar was found in $46 \%$ and $60 \%$ of patients reported from South Vietnam and Taiwan respectively.5,6 In the present case, the salient features of Scrub Typhus like fever, maculopapular rash, hepatosplenomegaly, pallor, myalgia, irritability were noted. However, no eschar was seen in this case. Complications like pneumonia, myocarditis, pericarditis, acute respiratory distress syndrome are seen; rarely meningitis, encephalitis, macrophage activation syndrome, acute renal failure can occur.

The present case had signs of meningeal involvement with altered sensorium \& later had anicteric hepatitis. Other organs were normal. Among the laboratory parameters, the most consistent abnormality noticed was elevation of liver enzymes, which was present in $95.9 \%$ of the cases. ${ }^{3}$ We considered the diagnosis of rickettsial encephalitis, thus patient was started on oral doxycycline and IV Chloramphenicol. Scrub typhus is known to produce serious complications and has a mortality rate of 7-30\%.6 Deaths are attributable to late presentation, delayed diagnosis and drug resistance. 
CSF abnormalities in Rickettsial meningitis is similar to that of viral or tuberculous meningitis. ${ }^{7}$ The features favouring Rickettsial meningitis were duration of fever more than five days, CSF pleocytosis of lower magnitude, CSF lymphocytes more than $50 \%$ and elevation of SGPT.

A Pondicherry based study had positive Weil Felix test in 39/50 patients in titers 1:80 or more. In two of these cases Weil Felix test was negative on admission, but when repeated in the convalescent period became positive. ${ }^{2}$ Weil Felix test has not been found to be a sensitive test to detect scrub typhus in the community by other studies also, but when positive, it is highly specific. ${ }^{8,9}$ Weil Felix test is usually positive during the second week of illness. This test is based on the detection of antibodies to various Proteus species which contain antigens with cross reacting epitopes to antigens from members of the genus Rickettsia. Positive test with OXK strain of Proteus mirabilis is suggestive of scrub typhus. Positive test with OX2 and OX19 strains of Proteus suggests infection by typhus and spotted fever groups of Rickettsiae.

Criteria suggested for the diagnosis of scrub typhus is a single titre of 1:320 or greater, or a fourfold rise in titre starting from 1:80 for OXK. A good correlation between the results of Weil Felix test and the detection of IgM antibodies by an immunofluorescence assay has been observed. According to Issac et al, from Christian Medical College, Vellore, the specificity of the test is high, even at a titer as low as $1 / 20 .{ }^{8}$ Hence, they suggested that patients with low titres also should be evaluated for scrub typhus. However the test lacks sensitivity. In a different study from the same institution which evaluated various serological tests for scrub typhus, Weil Felix test was found to have a sensitivity of only $43 \%$ but a specificity of $98 \%$ for titres $1: 80$ or more. ${ }^{9}$

Serological tests for Rickettsial diseases including the specific IgM antibody tests become positive only in the second week and a second sample at a later time is often required; serological tests cannot provide early diagnosis and a specific diagnosis may not be available until after the patient has died or recovered..$^{10}$ In our case Weil-Felix repeated in $3^{\text {rd }}$ week was inconclusive. Indirect fluorescent antibody (IFA) or immunoperoxidase assays were not done in our case. Doxycycline is the treatment of choice for scrub typhus. Highly virulent or potentially doxycycline-resistant 0 . tsutsugamushi strains have emerged in some regions of Thailand. Our patient had neurologic deterioration inspite of 3 days of doxycycline, hence IV Chloramphenicol was started. Child improved significantly on day3 of chloramphenicol.

Thus, clinical diagnosis and response to treatment are the best indicators of diagnosing scrub typhus. Scrub typhus must be considered in children presenting with subacute meningitis, fever, rash and hepatosplenomegaly.

\section{REFERENCES:}

1. K Saifudheen, K Sajeeth Kumar, James Jose et al. First case of scrub typhus with meningoencephalitis from Kerala: An emerging infectious threat. Ann Indian Acad Neurol. 2012 Apr-Jun; 15(2): 141-144.

2. M Vivekanandan, A Mani, YS Priya, AP Singh, S Jayakumar, S Purty. Outbreak of scrub typhus in Pondicherry. J Assoc Physicians India, 58 (2010), pp. 24-28.

3. Mathai E, Rolain JM, Verghese GM et al. Outbreak of Scrub Typhus in Southern India during the cooler months. Ann. New York Acad Sci. 2003; 990:359-64.

4. Sharma A, Mahajan S, Gupta ML et al. Investigation of an outbreak of Scrub Typhus in Himalayan region of India. Jpn J Infect Dis 2005;58: 208-10. 


\section{CASE REPORT}

5. Berman SJ, Kundin WD. Scrub Typhus in South Vietnam. A study of 87 cases. Ann Intern Med. 1973; 79:26-30.

6. Wang CC, Liu SF, Liu JW, et al. Acute Respiratory distress syndrome in Scrub Typhus. Am J Trop Med Hyg 2007; 76: 1148-52.

7. Silpapojakul K, Ukkachoke C, Krisanapan S. Rickettsial Meningitis and encephalitis. Arch Intern Med 1991; 151:1753-1757.

8. Issac R, Varghese GM, Mathai E et al. Scrub Typhus: Prevalence and diagnostic issues in rural Southern India. Clin Infect Dis 2004;39: 1395-6.

9. Prakash JA, Abraham OC, Mathai E. Evaluation of tests for serological diagnosis of scrub typhus. Trop Doct 2006; 36: 212-3.

10. Kamarasu K, Mathan M, Rajagopal V, Subramaniam K et al. Serological evidence for wide distribution of spotted fevers and scrub typhus fever in Tamil Nadu. Indian J Med Res 126: 2007;128-130.

\section{AUTHORS:}

1. Swati M. Gadappa

2. Deepika Chopra

\section{PARTICULARS OF CONTRIBUTORS:}

1. Assistant Professor, Department of Paediatrics, Bharati Vidyapeeth Medical College, Pune.

2. Post Graduate Resident, Department of Paediatrics, Bharati Vidyapeeth Medical College, Pune.

\section{NAME ADDRESS EMAIL ID OF THE}

\section{CORRESPONDING AUTHOR:}

Dr. Swati Gadappa,

601/B3, Runwal Seagull Township,

Handewadi Road,

Hadapsar, Pune-411028.

Email: swatigadappa@yahoo.co.in

Date of Submission: 04/11/2014.

Date of Peer Review: 05/11/2014.

Date of Acceptance: 06/11/2014.

Date of Publishing: 10/11/2014. 